\title{
ON HYPONORMALITY OF TOEPLITZ OPERATORS WITH POLYNOMIAL AND SYMMETRIC TYPE SYMBOLS
}

\author{
Munmun Hazarika And Ambeswar Phukon
}

\begin{abstract}
In [6], it was shown that hyponormality for Toeplitz operators with polynomial symbols can be reduced to classical Schur's algorithm in function theory. In [6], Zhu has also given the explicit values of the Schur's functions $\Phi_{0}, \Phi_{1}$ and $\Phi_{2}$. Here we explicitly evaluate the Schur's function $\Phi_{3}$. Using this value we find necessary and sufficient conditions under which the Toeplitz operator $T_{\varphi}$ is hyponormal, where $\varphi$ is a trigonometric polynomial given by $\varphi(z)=\sum_{n=-N}^{N} a_{n} z_{n}(N \geq 4)$ and satisfies the condition $\bar{a}_{N}\left(\begin{array}{c}a_{-1} \\ a_{-2} \\ a_{-4} \\ \vdots \\ a_{-N}\end{array}\right)=a_{-N}\left(\begin{array}{c}\bar{a}_{1} \\ \bar{a}_{2} \\ \bar{a}_{4} \\ \vdots \\ \bar{a}_{N}\end{array}\right)$. Finally we illustrate the easy applicability of the derived results with a few examples.
\end{abstract}

\section{Introduction}

A bounded linear operator $T$ on a Hilbert space is said to be hyponormal if its self commutator $\left[T^{*}, T\right]:=T^{*} T-T T^{*}$ is positive semi definite. Given $\varphi \in L^{\infty}(\mathbb{T})$, the Toeplitz operator with symbol $\varphi$ is the operator $T_{\varphi}$ on the Hardy space $H^{2}(\mathbb{T})$ of the unit circle $\mathbb{T}=\partial \mathbb{D}$ defined by $T_{\varphi} f:=P(\varphi . f)$, where $f \in H^{2}(\mathbb{T})$ and $P$ denotes the orthogonal projection that maps $L^{2}(\mathbb{T})$ onto $H^{2}(\mathbb{T})$.

We are interested in the following question: "if $\varphi$ is a trigonometric polynomial, then when is the trigonometric Toeplitz operator $T_{\varphi}$ hyponormal?" If $\varphi$ is a trigonometric polynomial of the form $\varphi(z)=\sum_{n=-m}^{N} a_{n} z^{n}$ where $a_{-m}$ and $a_{N}$ are non zero, then it was shown in [4] and [2] that hyponormality of $T_{\varphi}$ implies $m \leq N$ and $\left|a_{-m}\right| \leq\left|a_{N}\right|$. In [2] it was shown that for $\varphi(z)=\sum_{n=-m}^{N} a_{n} z^{n}$, if $\left|a_{-m}\right|=\left|a_{N}\right| \neq 0$, then $T_{\varphi}$ is hyponormal if and only

Received October 19, 2009.

2010 Mathematics Subject Classification. Primary 47B35, 47B20.

Key words and phrases. Toeplitz operators, hyponormal operators, trigonometric polynomial.

This work was financially supported by DST SR/FTP/MS-13/2008 and UGC F. 540/TF/2007(NERO)/476. 
if the coefficients of $\varphi$ satisfy the following 'symmetry' condition:

$$
\bar{a}_{N}\left(\begin{array}{c}
a_{-1} \\
a_{-2} \\
\vdots \\
a_{-m}
\end{array}\right)=a_{-m}\left(\begin{array}{c}
\bar{a}_{N-m+1} \\
\bar{a}_{N-m+2} \\
\vdots \\
\bar{a}_{N}
\end{array}\right)
$$

But if $\left|a_{-m}\right| \neq\left|a_{N}\right|$, then the case of arbitrary polynomial $\varphi$, though solved in principle by Cowen's Theorem [1] or Zhu's Theorem [6], is in practice very complicated. In [5], the hyponormality of $T_{\varphi}$ was studied when $\varphi(z)=$ $\sum_{n=-N}^{N} a_{n} z^{n}$ satisfies a 'partial' symmetry condition:

$$
\bar{a}_{N}\left(\begin{array}{c}
a_{-m} \\
a_{-(m+1)} \\
\vdots \\
a_{-N}
\end{array}\right)=a_{-N}\left(\begin{array}{c}
\bar{a}_{m} \\
\bar{a}_{m+1} \\
\vdots \\
\bar{a}_{N}
\end{array}\right) \text { for } m-1 \leq \frac{N}{2} .
$$

The authors gave in the paper a necessary and sufficient condition for the hyponormality of $T_{\varphi}$ when $\varphi$ satisfies (1) and has the property $\mid \bar{a}_{N} a_{-(m-1)}-$ $\left.a_{-N} \bar{a}_{m-1}|=| a_{N}\right|^{2}-\left|a_{-N}\right|^{2}$. Further, in [3] we find a complete criterion for hyponormality of $T_{\varphi}$ when $\varphi$ satisfies (1).

In this paper, we consider the trigonometric polynomial $\varphi(z)=\sum_{n=-N}^{N} a_{n} z^{n}$ with $\left|a_{-N}\right|<\left|a_{N}\right|$ such that

$$
\bar{a}_{N}\left(\begin{array}{c}
a_{-1} \\
a_{-2} \\
a_{-4} \\
\vdots \\
a_{-N}
\end{array}\right)=a_{-N}\left(\begin{array}{c}
\bar{a}_{1} \\
\bar{a}_{2} \\
\bar{a}_{4} \\
\vdots \\
\bar{a}_{N}
\end{array}\right) \text { and } \bar{a}_{N} a_{-3} \neq a_{-N} \bar{a}_{3}
$$

and we give necessary and sufficient conditions for hyponormality of $T_{\varphi}$.

\section{Evaluation of Schur's function $\Phi_{3}$}

In [6], Kehe Zhu applied Schur's algorithm to the Schur's function $\Phi_{N}$ in order to determine hyponormality of Toeplitz operators with polynomial symbols. We begin the section with the brief description of Zhu's idea.

Suppose that $f(z)=\sum_{j=0}^{\infty} c_{j} z^{j}$ is in the closed unit ball of $H^{\infty}(\mathbb{T})$. If $f_{0}=f$, define by induction a sequence $\left\{f_{n}\right\}$ of functions in the closed unit ball of $H^{\infty}(\mathbb{T})$ as follows:

$$
f_{n+1}(z)=\frac{f_{n}(z)-f_{n}(0)}{z\left(1-\overline{f_{n}(0)} f_{n}(z)\right)},|z|<1, \quad n=0,1,2, \ldots
$$

We write $f_{n}(0)=\Phi_{n}\left(c_{0}, \ldots, c_{n}\right), n=0,1,2, \ldots$, where $\Phi_{n}$ is a function of $n+1$ complex variables. We call the $\Phi_{n}$ 's Schur's functions. Zhu's theorem can now be written as follows: 
Theorem 2.1 ([6]). If $\varphi(z)=\sum_{n=-N}^{N} a_{n} z^{n}$, where $a_{N} \neq 0$ and if

$$
\left(\begin{array}{c}
\overline{c_{0}} \\
\overline{c_{1}} \\
\vdots \\
\bar{c}_{N-1}
\end{array}\right)=\left(\begin{array}{ccccc}
a_{1} & a_{2} & \cdots & a_{N-1} & a_{N} \\
a_{2} & a_{3} & \cdots & a_{N} & 0 \\
\vdots & \vdots & \ddots & \vdots & \vdots \\
a_{N} & 0 & \cdots & 0 & 0
\end{array}\right)^{-1}\left(\begin{array}{c}
\bar{a}_{-1} \\
\bar{a}_{-2} \\
\vdots \\
\bar{a}_{-N}
\end{array}\right)
$$

then $T_{\varphi}$ is hyponormal if and only if $\left|\Phi_{n}\left(c_{0}, \ldots, c_{n}\right)\right| \leq 1$ for each $n=0,1, \ldots$, $N-1$.

Note that each $\Phi_{n}\left(c_{0}, \ldots, c_{n}\right)$ is a rational function of the form

$$
\Phi_{n}\left(c_{0}, \ldots, c_{n}\right)=\frac{F_{n}\left(c_{0}, \ldots, c_{n}\right)}{G_{n}\left(c_{0}, \ldots, c_{n}\right)},
$$

where $F_{n}$ and $G_{n}$ are polynomials. Thus the inequalities $\left|\Phi_{n}\left(c_{0}, \ldots, c_{n}\right)\right| \leq 1$ should be understood as $\left|F_{n}\left(c_{0}, \ldots, c_{n}\right)\right| \leq\left|G_{n}\left(c_{0}, \ldots, c_{n}\right)\right|$.

In [6] Zhu also listed the first three Schur's functions as follows:

$$
\begin{aligned}
\Phi_{0}\left(c_{0}\right) & =c_{0}, \\
\Phi_{1}\left(c_{0}, c_{1}\right) & =\frac{c_{1}}{1-\left|c_{0}\right|^{2}}, \\
\Phi_{2}\left(c_{0}, c_{1}, c_{2}\right) & =\frac{c_{2}\left(1-\left|c_{0}\right|^{2}\right)+\overline{c_{0}} c_{1}^{2}}{\left(1-\left|c_{0}\right|^{2}\right)^{2}-\left|c_{1}\right|^{2}} .
\end{aligned}
$$

Here in this section we evaluate $\Phi_{3}\left(c_{0}, c_{1}, c_{2}, c_{3}\right)$ using the Schur's algorithm, and we get,

$$
\begin{aligned}
& \Phi_{3}\left(c_{0}, c_{1}, c_{2}, c_{3}\right) \\
= & \frac{\left(\left(1-\left|c_{0}\right|^{2}\right)^{2}-\left|c_{1}\right|^{2}\right)\left(\left(1-\left|c_{0}\right|^{2}\right) c_{3}+\overline{c_{0}} c_{1} c_{2}\right)+\left(c_{2}\left(1-\left|c_{0}\right|^{2}\right)+\overline{c_{0}} c_{1}^{2}\right)\left(\overline{c_{0}}\left(1-\left|c_{0}\right|^{2}\right) c_{1}+\overline{c_{1}} c_{2}\right)}{\left(\left(1-\left|c_{0}\right|^{2}\right)^{2}-\left|c_{1}\right|^{2}\right)^{2}-\left|c_{2}\left(1-\left|c_{0}\right|^{2}\right)+\overline{c_{0} c_{1}}\right|^{2}} .
\end{aligned}
$$

We briefly outline the steps leading to this result. For the function $f(z)=$ $\sum_{j=0}^{\infty} c_{j} z^{j}$ in the closed unit ball of $H^{\infty}(\mathbb{T})$ :

$\operatorname{Step}(1): f_{0}(z)=f(z)$ and so $\Phi_{0}\left(c_{0}\right)=f_{0}(0)=f(0)=c_{0}$.

$\operatorname{Step}(2): f_{1}(z)=\frac{f_{0}(z)-f_{0}(0)}{z\left(1-\overline{f_{0}(0)} f_{0}(z)\right)}=\frac{\sum_{k=1}^{\infty} c_{k} z^{k-1}}{1-\left|c_{0}\right|^{2}-\overline{c_{0}} \sum_{k=1}^{\infty} c_{k} z^{k}}$ and so $\Phi_{1}\left(c_{0}, c_{1}\right)=$ $f_{1}(0)=\frac{c_{1}}{1-\left|c_{0}\right|^{2}}$.

$\operatorname{Step}(3): f_{2}(z)=\frac{f_{1}(z)-f_{1}(0)}{z\left(1-\overline{f_{1}(0)} f_{1}(z)\right)}=\frac{N}{z D}$, where

$$
\begin{aligned}
N & =\frac{\left(1-\left|c_{0}\right|^{2}\right) \sum_{k=2}^{\infty} c_{k} z^{k-1}+\overline{c_{0}} c_{1} \sum_{k=1}^{\infty} c_{k} z^{k}}{\left(1-\overline{c_{0}} \sum_{k=0}^{\infty} c_{k} z^{k}\right)\left(1-\left|c_{0}\right|^{2}\right)}, \\
D & =\frac{\left(\left(1-\left|c_{0}\right|^{2}\right)^{2}-\left|c_{1}\right|^{2}\right)-\overline{c_{0}}\left(1-\left|c_{0}\right|^{2}\right) \sum_{k=1}^{\infty} c_{k} z^{k}-\overline{c_{1}} \sum_{k=2}^{\infty} c_{k} z^{k-1}}{\left(1-\overline{c_{0}} \sum_{k=0}^{\infty} c_{k} z^{k}\right)\left(1-\left|c_{0}\right|^{2}\right)} .
\end{aligned}
$$

Therefore,

$$
f_{2}(z)=\frac{\left(1-\left|c_{0}\right|^{2}\right) \sum_{k=2}^{\infty} c_{k} z^{k-2}+\overline{c_{0}} c_{1} \sum_{k=1}^{\infty} c_{k} z^{k-1}}{\left(\left(1-\left|c_{0}\right|^{2}\right)^{2}-\left|c_{1}\right|^{2}\right)-\overline{c_{0}}\left(1-\left|c_{0}\right|^{2}\right) \sum_{k=1}^{\infty} c_{k} z^{k}-\overline{c_{1}} \sum_{k=2}^{\infty} c_{k} z^{k-1}}
$$


and

$\operatorname{Step}(4)$ :

$$
\Phi_{2}\left(c_{0}, c_{1}, c_{2}\right)=f_{2}(0)=\frac{c_{2}\left(1-\left|c_{0}\right|^{2}\right)+\overline{c_{0}} c_{1}^{2}}{\left(1-\left|c_{0}\right|^{2}\right)^{2}-\left|c_{1}\right|^{2}} .
$$

where

$$
f_{3}(z)=\frac{f_{2}(z)-f_{2}(0)}{z\left(1-\overline{f_{2}(0)} f_{2}(z)\right)}=\frac{N}{D}
$$

$$
\begin{aligned}
N= & \left(\left(1-\left|c_{0}\right|^{2}\right)^{2}-\left|c_{1}\right|^{2}\right)\left(\left(1-\left|c_{0}\right|^{2}\right) \sum_{k=2}^{\infty} c_{k} z^{k-2}+\bar{c}_{0} c_{1} \sum_{k=1}^{\infty} c_{k} z^{k-1}\right) \\
& -\left(c_{2}\left(1-\left|c_{0}\right|^{2}\right)+\overline{c_{0}} c_{1}^{2}\right)\left(\left(\left(1-\left|c_{0}\right|^{2}\right)^{2}-\left|c_{1}\right|^{2}\right)-\overline{c_{0}}\left(1-\left|c_{0}\right|^{2}\right) \sum_{k=1}^{\infty} c_{k} z^{k}-\overline{c_{1}} \sum_{k=2}^{\infty} c_{k} z^{k-1}\right)
\end{aligned}
$$

and

$$
\begin{aligned}
D= & \left(\left(1-\left|c_{0}\right|^{2}\right)^{2}-\left|c_{1}\right|^{2}\right)\left(\left(\left(1-\left|c_{0}\right|^{2}\right)^{2}-\left|c_{1}\right|^{2}\right)-\overline{c_{0}}\left(1-\left|c_{0}\right|^{2}\right) \sum_{k=1}^{\infty} c_{k} z^{k}-\overline{c_{1}} \sum_{k=2}^{\infty} c_{k} z^{k-1}\right) \\
& -\left(\bar{c}_{2}\left(1-\left|c_{0}\right|^{2}\right)+c_{0} \bar{c}_{1}^{2}\right)\left(\left(1-\left|c_{0}\right|^{2}\right) \sum_{k=2}^{\infty} c_{k} z^{k-2}+\overline{c_{0}} c_{1} \sum_{k=1}^{\infty} c_{k} z^{k-1}\right) .
\end{aligned}
$$

Thus,

$$
\begin{aligned}
& \Phi_{3}\left(c_{0}, c_{1}, c_{2}, c_{3}\right) \\
= & f_{3}(0) \\
= & \frac{\left(\left(1-\left|c_{0}\right|^{2}\right)^{2}-\left|c_{1}\right|^{2}\right)\left(\left(1-\left|c_{0}\right|^{2}\right) c_{3}+\overline{c_{0}} c_{1} c_{2}\right)+\left(c_{2}\left(1-\left|c_{0}\right|^{2}\right)+\overline{c_{0}} c_{1}^{2}\right)\left(\overline{c_{0}}\left(1-\left|c_{0}\right|^{2}\right) c_{1}+\overline{c_{1}} c_{2}\right)}{\left(\left(1-\left|c_{0}\right|^{2}\right)^{2}-\left|c_{1}\right|^{2}\right)^{2}-\left|c_{2}\left(1-\left|c_{0}\right|^{2}\right)+\overline{c_{0} c_{1}{ }^{2}}\right|^{2}} .
\end{aligned}
$$

\section{Hyponormality condition}

Theorem 3.1. Suppose $\varphi(z)=\sum_{n=-N}^{N} a_{n} z^{n}$ is such that

$$
\bar{a}_{N}\left(\begin{array}{c}
a_{-1} \\
a_{-2} \\
a_{-4} \\
\vdots \\
a_{-N}
\end{array}\right)=a_{-N}\left(\begin{array}{c}
\bar{a}_{1} \\
\bar{a}_{2} \\
\bar{a}_{4} \\
\vdots \\
\bar{a}_{N}
\end{array}\right) \text { and } \alpha:=\frac{\operatorname{det}\left(\begin{array}{cc}
a_{-3} & a_{-N} \\
\bar{a}_{3} & \bar{a}_{N}
\end{array}\right)}{\left|a_{N}\right|^{2}-\left|a_{-N}\right|^{2}} .
$$

(1) If $N=4$, then $T_{\varphi}$ is hyponormal if and only if
(a) $|\alpha| \leq 1$
(b) $\left|\bar{\alpha} a_{-4}-a_{3}\right| \leq\left|a_{4}\right|\left(\frac{1}{|\alpha|}-|\alpha|\right)$,
(c) $\left|\left(1-|\alpha|^{2}\right)\left(\bar{a}_{3}^{2}-\bar{a}_{2} \bar{a}_{4}-\bar{a}_{3} \bar{a}_{-4} \alpha\right)+\alpha\left(\bar{a}_{-4} \alpha-\bar{a}_{3}\right)\left(\bar{a}_{-4}-\bar{a}_{3} \bar{\alpha}\right)\right|$$$
\leq|\alpha|\left(\left|a_{4}\right|^{2}\left(\frac{1}{|\alpha|}-|\alpha|\right)^{2}-\left|a_{-4} \bar{\alpha}-a_{3}\right|^{2}\right)
$$

(2) If $N \geq 5$, then $T_{\varphi}$ is hyponormal if and only if
(a) $|\alpha| \leq 1$,
(b) $\left|\frac{a_{N-1}}{a_{N}}\right| \leq \frac{1}{|\alpha|}-|\alpha|$, 


$$
\text { (c) }\left|\frac{1}{\alpha}\left(\frac{a_{N-1}}{a_{N}}\right)^{2}-\left(\frac{1}{\alpha}-\bar{\alpha}\right) \frac{a_{N-2}}{a_{N}}\right| \leq\left(\frac{1}{|\alpha|}-|\alpha|\right)^{2}-\left|\frac{a_{N-1}}{a_{N}}\right|^{2} \text {. }
$$

Remark 3.2. Here we assume that $\operatorname{det}\left(\begin{array}{cc}a_{-3} & a_{-N} \\ \bar{a}_{3} & \bar{a}_{N}\end{array}\right)$ and $\left|a_{N}\right|^{2}-\left|a_{-N}\right|^{2}$ are nonzero, because otherwise the results follow from [2] and [5]. Thus, $a_{-3} \bar{a}_{N} \neq$ $a_{-N} \bar{a}_{3}$ and $\left|a_{-N}\right|<\left|a_{N}\right|$.

Proof. (1) When $N=4$.

Using Proposition $1[5], c_{0}, c_{1}, c_{2}, c_{3}$ are determined as follows:

$$
\begin{aligned}
& c_{0}=\frac{a_{-4}}{\bar{a}_{4}}, \\
& c_{1}=\left(\bar{a}_{4}\right)^{-1}\left(a_{-3}-c_{0} \bar{a}_{3}\right), \\
& c_{2}=\left(\bar{a}_{4}\right)^{-1}\left(a_{-2}-c_{0} \bar{a}_{2}-c_{1} \bar{a}_{3}\right), \\
& c_{3}=\left(\bar{a}_{4}\right)^{-1}\left(a_{-1}-c_{0} \bar{a}_{1}-c_{1} \bar{a}_{2}-c_{2} \bar{a}_{3}\right) .
\end{aligned}
$$

Calculating and simplifying we get

$$
\begin{aligned}
c_{1} & =\left(\bar{a}_{4}\right)^{-2}\left(\left|a_{4}\right|^{2}-\left|a_{-4}\right|^{2}\right) \alpha, \\
c_{2} & =\left(\bar{a}_{4}\right)^{-1}\left(a_{-2}-\frac{a_{-4}}{\bar{a}_{4}} \bar{a}_{2}-c_{1} \bar{a}_{3}\right)=-\frac{c_{1} \bar{a}_{3}}{\bar{a}_{4}}, \\
c_{3} & =\left(\frac{\bar{a}_{3}^{2}-\bar{a}_{2} \bar{a}_{4}}{\bar{a}_{4}^{2}}\right) c_{1}, \\
1-\left|c_{0}\right|^{2} & =1-\frac{\left|a_{-4}\right|^{2}}{\left|a_{4}\right|^{2}}=\left|a_{4}\right|^{-2}\left(\left|a_{4}\right|^{2}-\left|a_{-4}\right|^{2}\right), \\
\frac{\left|c_{1}\right|}{1-\left|c_{0}\right|^{2}} & =|\alpha| .
\end{aligned}
$$

We also have,

$$
\begin{aligned}
\Phi_{0} & =c_{0}, \\
\Phi_{1} & =\frac{c_{1}}{1-\left|c_{0}\right|^{2}}, \\
\Phi_{2} & =\frac{c_{2}\left(1-\left|c_{0}\right|^{2}\right)+\overline{c_{0}} c_{1}^{2}}{\left(1-\left|c_{0}\right|^{2}\right)^{2}-\left|c_{1}\right|^{2}}
\end{aligned}
$$

$\Phi_{3}=\frac{\left(\left(1-\left|c_{0}\right|^{2}\right)^{2}-\left|c_{1}\right|^{2}\right)\left(\left(1-\left|c_{0}\right|^{2}\right) c_{3}+\overline{c_{0}} c_{1} c_{2}\right)+\left(c_{2}\left(1-\left|c_{0}\right|^{2}\right)+\overline{c_{0}} c_{1}{ }^{2}\right)\left(\overline{c_{0}}\left(1-\left|c_{0}\right|^{2}\right) c_{1}+\overline{c_{1} c_{2}}\right)}{\left(\left(1-\left|c_{0}\right|^{2}\right)^{2}-\left|c_{1}\right|^{2}\right)^{2}-\left|c_{2}\left(1-\left|c_{0}\right|^{2}\right)+\overline{c_{0} c_{1}}\right|^{2}}$.

Using expressions (6) to (10) in (11), (12), (13) and (14) and simplifying, we get

$\left|\Phi_{0}\right| \leq 1$ if and only if $\left|a_{-4}\right| \leq\left|a_{4}\right|$,

$\left|\Phi_{1}\right| \leq 1$ if and only if $|\alpha| \leq 1$,

$\left|\Phi_{2}\right| \leq 1$ if and only if $\left|\bar{a}_{-4} \alpha-\bar{a}_{3}\right| \leq\left|a_{4}\right|\left(\frac{1}{|\alpha|}-|\alpha|\right)$, 
$\left|\Phi_{3}\right| \leq 1$ if and only if

$$
\begin{aligned}
& \left|\left(1-|\alpha|^{2}\right)\left(\bar{a}_{3}^{2}-\bar{a}_{2} \bar{a}_{4}-\bar{a}_{3} \bar{a}_{-4} \alpha\right)+\alpha\left(\bar{a}_{-4} \alpha-\bar{a}_{3}\right)\left(\bar{a}_{-4}-\bar{a}_{3} \bar{\alpha}\right)\right| \\
\leq & |\alpha|\left(\left|a_{4}\right|^{2}\left(\frac{1}{|\alpha|}-|\alpha|\right)^{2}-\left|\bar{a}_{-4} \alpha-\bar{a}_{3}\right|^{2}\right) .
\end{aligned}
$$

The result follows from Theorem 2.1.

(2) When $N>4$

Let $c_{0}, c_{1}, \ldots, c_{N-1}$ be determined by Proposition 1 [5]. Then,

$$
\begin{aligned}
c_{0} & =\frac{a_{-N}}{\bar{a}_{N}}, \\
c_{1} & =\left(\bar{a}_{N}\right)^{-1}\left(a_{-(N-1)}-c_{0} \bar{a}_{N-1}\right)=\left(\bar{a}_{N}\right)^{-2}\left(\bar{a}_{N} a_{-(N-1)}-a_{-N} \bar{a}_{N-1}\right)=0, \\
& \vdots \\
c_{N-4} & =\left(\bar{a}_{N}\right)^{-1}\left(a_{-4}-c_{0} \bar{a}_{4}-c_{1} \bar{a}_{5}-\ldots-c_{N-5} \bar{a}_{N-1}\right)=0, \\
c_{N-3}= & \left(\bar{a}_{N}\right)^{-1}\left(a_{-3}-c_{0} \bar{a}_{3}-c_{1} \bar{a}_{4}-\ldots-c_{N-4} \bar{a}_{N-1}\right) \\
& =\left(\bar{a}_{N}\right)^{-2}\left(a_{-3} \bar{a}_{N}-a_{-N} \bar{a}_{3}\right), \\
c_{N-2}= & \left(\bar{a}_{N}\right)^{-1}\left(a_{-2}-c_{0} \bar{a}_{2}-c_{1} \bar{a}_{3}-c_{2} \bar{a}_{4}-\ldots-c_{N-3} \bar{a}_{N-1}\right), \\
& =-\left(\frac{\bar{a}_{N-1}}{\bar{a}_{N}}\right)_{N-3}, \\
c_{N-1}= & \left(\bar{a}_{N}\right)^{-1}\left(a_{-1}-c_{0} \bar{a}_{1}-c_{1} \bar{a}_{2}-\ldots-c_{N-3} \bar{a}_{N-2}-c_{N-2} \bar{a}_{N-1}\right) \\
= & \left(\bar{a}_{N}\right)^{-1}\left(-c_{N-1} \bar{a}_{N-2}+\frac{\bar{a}_{N-1}}{\bar{a}_{N}} c_{N-3} \bar{a}_{N-1}\right) \\
= & \left(\left(\frac{\bar{a}_{N-1}}{\bar{a}_{N}}\right)^{2}-\frac{\bar{a}_{N-2}}{\bar{a}_{N}}\right) c_{N-3} .
\end{aligned}
$$

Thus, $b_{p}(z)=c_{0}+c_{N-3} z^{N-3}+c_{N-2} z^{N-2}+c_{N-1} z^{N-1}$ is the unique analytic polynomial of degree less than $N$ satisfying $\varphi-b_{p} \bar{\varphi} \in H^{\infty}$. By our assumption we have $c_{N-3} \neq 0$. Thus, by Proposition $3[5]$,

$$
\begin{aligned}
\Phi_{0} & =c_{0}, \\
\Phi_{1} & =\Phi_{2}=\ldots=\Phi_{N-4}=0, \\
\Phi_{N-3} & =\frac{c_{N-3}}{1-\left|c_{0}\right|^{2}}, \\
\Phi_{N-2} & =\frac{c_{N-2}}{\left(1-\left|c_{0}\right|^{2}\right)\left(1-\left|\Phi_{N-3}\right|^{2}\right)}, \\
\Phi_{N-1} & =\frac{\left(1-\left|\Phi_{N-3}\right|^{2}\right) c_{N-1} c_{N-3}+\left|\Phi_{N-3}\right|^{2} c_{N-2}^{2}}{c_{N-3}\left(1-\left|c_{0}\right|^{2}\right)\left(1-\left|\Phi_{N-3}\right|^{2}\right)^{2}\left(1-\left|\Phi_{N-2}\right|^{2}\right)} .
\end{aligned}
$$

Computing and simplifying from these relations, we get, 
$\left|\Phi_{0}\right| \leq 1$ if and only if $\left|a_{-N}\right| \leq\left|a_{N}\right|$, which is always true since we are taking $\left|a_{-N}\right|<\left|a_{N}\right|$,

$\left|\Phi_{N-3}\right| \leq 1$ if and only if $|\alpha| \leq 1$,

$\left|\Phi_{N-2}\right| \leq 1$ if and only if $\left|\frac{a_{N-1}}{a_{N}}\right| \leq \frac{1}{|\alpha|}-|\alpha|$,

$\left|\Phi_{N-1}\right| \leq 1$ if and only if $\left|\frac{1}{\alpha}\left(\frac{a_{N-1}}{a_{N}}\right)^{2}-\left(\frac{1}{\alpha}-\bar{\alpha}\right) \frac{a_{N-2}}{a_{N}}\right| \leq\left(\frac{1}{|\alpha|}-|\alpha|\right)^{2}-$ $\left|\frac{a_{N-1}}{a_{N}}\right|^{2}$.

The result follows from Theorem 2.1.

\section{Examples}

Example 1. For $\varphi(z)=z^{-4}+\lambda z^{-3}+2 z^{-1}+4 z^{3}+2 z^{6}$ we want to determine the values of $\lambda$ for which the Toeplitz operator $T_{\varphi}$ is hyponormal.

By Proposition 2 [5], $T_{\varphi}$ is hyponormal if and only if $T_{\psi}$ is hyponormal where $\psi(z)=z^{-4}+\lambda z^{-3}+2 z^{-1}+4 z+2 z^{4}$. Expressing $\psi(z)$ as $\sum_{n=-N}^{N} a_{n} z^{n}$ and comparing with the given expression, we have, $N=4$ and also $\bar{a}_{4}\left(\begin{array}{l}a_{-1} \\ a_{-2} \\ a_{-4}\end{array}\right)=$ $a_{-4}\left(\begin{array}{c}\bar{a}_{1} \\ \bar{a}_{2} \\ \bar{a}_{4}\end{array}\right)$. Hence, we can apply Case 1 of Theorem 3.1 to determine the values of $\lambda$ for which $T_{\psi}$ will be hyponormal. It may be noted that if $\lambda=0$, then $T_{\psi}$ is hyponormal by Theorem $1.4[2]$.

As $\alpha=\left|\begin{array}{cc}a_{-3} & a_{-4} \\ \bar{a}_{3} & \bar{a}_{4}\end{array}\right| /\left(\left|a_{4}\right|^{2}-\left|a_{-4}\right|^{2}\right)=\frac{2 \lambda}{3}$, thus refering to the notations used in Theorem 3.1 we have,

(i) $|\alpha| \leq 1$ if and only if $|\lambda| \leq \frac{3}{2}=1.5$,

(ii) $\left|\bar{\alpha} a_{-4}-a_{3}\right| \leq\left|a_{4}\right|\left(\frac{1}{|\alpha|}-|\alpha|\right)$ if and only if $|\lambda| \leq \sqrt{\frac{3}{2}}=1.22$, correct upto two decimal places,

(iii)

$$
\begin{aligned}
& \left|\left(1-|\alpha|^{2}\right)\left(\bar{a}_{3}^{2}-\bar{a}_{2} \bar{a}_{4}-\bar{a}_{3} \bar{a}_{-4} \alpha\right)+\alpha\left(\bar{a}_{-4} \alpha-\bar{a}_{3}\right)\left(\bar{a}_{-4}-\bar{a}_{3} \bar{\alpha}\right)\right| \\
\leq & |\alpha|\left(\left|a_{4}\right|^{2}\left(\frac{1}{|\alpha|}-|\alpha|\right)^{2}-\left|a_{-4} \bar{\alpha}-a_{3}\right|^{2}\right) \\
& \text { if and only if } 12|\lambda|^{4}-6|\lambda|^{3}-72|\lambda|^{2}+81 \geq 0 \\
& \text { if and only if }|\lambda| \leq 1.13 .
\end{aligned}
$$

This can be seen from the graph of $12|\lambda|^{4}-6|\lambda|^{3}-72|\lambda|^{2}+81=0$ in Figure 1:

Thus, $T_{\varphi}$ is hyponormal if and only if $|\lambda| \leq 1.13$ (correct upto two decimal places).

Example 2. If $\varphi(z)=z^{-4}+\lambda z^{-3}+z^{-2}+2 z^{-1}+1+4 z+2 z^{2}+2 z^{4}$, then using Theorem 3.1 (Case 1) as in Example 1, we get that the Toeplitz operator $T_{\varphi}$ is hyponormal if and only if $|\lambda| \leq 0.95$ (correct upto two decimal places). 


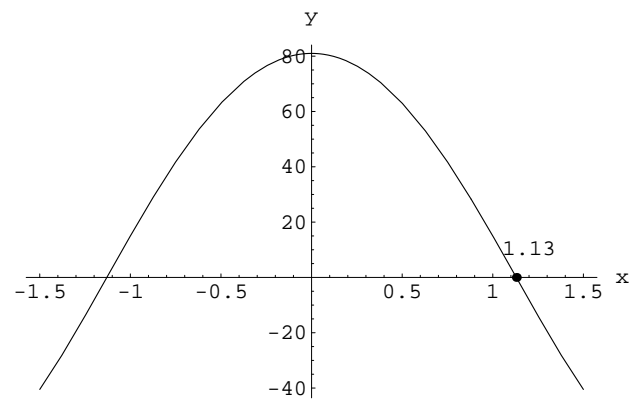

Figure 1

Example 3. If $\varphi(z)=2 z^{-5}+2 z^{-4}+\lambda z^{-3}+4 z^{-2}+2 z^{-1}+3 z+6 z^{2}+3 z^{3}+$ $3 z^{4}+3 z^{5}$, then using Theorem 3.1 (Case 2) we get that the Toeplitz operator $T_{\varphi}$ is hyponormal if and only if $0.34 \leq \lambda \leq 0.58$ (correct upto two decimal places).

Example 4 . If $\varphi(z)=z^{-6}+3 z^{-5}+z^{-4}+2 z^{-2}+z^{-1}+2 z+4 z^{2}+\lambda z^{3}+2 z^{4}+$ $6 z^{5}+2 z^{6}$, then using Theorem 3.1 (Case 2) we get that the Toeplitz operator $T_{\varphi}$ is hyponormal if and only if $|\lambda| \leq 0.32$ (correct upto two decimal places).

\section{Conclusion}

If for $N \geq 4$ and $\varphi(z)=\sum_{n=-N}^{N} a_{n} z^{n}$ with $\left|a_{N}\right|>\left|a_{-N}\right|$, we have

$$
\bar{a}_{N}\left(\begin{array}{c}
a_{-1} \\
a_{-2} \\
a_{-4} \\
\vdots \\
a_{-N}
\end{array}\right)=a_{-N}\left(\begin{array}{c}
\bar{a}_{1} \\
\bar{a}_{2} \\
\bar{a}_{4} \\
\vdots \\
\bar{a}_{N}
\end{array}\right) \text { and } \bar{a}_{N} a_{-3} \neq a_{-N} \bar{a}_{3}
$$

Then for $N \geq 6$ we can also apply Theorem 1 [3]. Particularly, for very large $N$, application of Theorem 1 [3] would significantly reduce the work load in determining the hyponormality of $T_{\varphi}$. However, this theorem do not give a ready set of conditions to finally determine hyponormality. In view of this, Theorem 3.1 offers an alternative method to determine hyponormality of $T_{\varphi}$ under the given restrictions on $\varphi$.

For $N \geq 3$, if

$$
\bar{a}_{N}\left(\begin{array}{c}
a_{-1} \\
a_{-3} \\
\vdots \\
a_{-N}
\end{array}\right)=a_{-N}\left(\begin{array}{c}
\bar{a}_{1} \\
\bar{a}_{3} \\
\vdots \\
\bar{a}_{N}
\end{array}\right) \text { and } \bar{a}_{N} a_{-2} \neq a_{-N} \bar{a}_{2}
$$

then Theorem 8 [5] gives a similar alternative method to determine hyponormality of $T_{\varphi}$. 


\section{References}

[1] C. C. Cowen, Hyponormality of Toeplitz operators, Proc. Amer. Math. Soc. 103 (1988), no. 3, 809-812.

[2] D. R. Farenick and W. Y. Lee, Hyponormality and spectra of Toeplitz operators, Trans. Amer. Math. Soc. 348 (1996), no. 10, 4153-4174.

[3] I. S. Hwang and W. Y. Lee, Hyponormality of Toeplitz operators with polynomial and symmetric-type symbols, Integral Equations Operator Theory 50 (2004), no. 3, 363-373.

[4] T. Ito and T. K. Wong, Subnormality and quasinormality of Toeplitz operators, Proc. Amer. Math. Soc. 34 (1972), 157-164.

[5] I. H. Kim and W. Y. Lee, On hyponormal Toeplitz operators with polynomial and symmetric-type symbols, Integral Equations Operator Theory 32 (1998), no. 2, 216-233.

[6] K. Zhu, Hyponormal Toeplitz operators with polynomial symbols, Integral Equations Operator Theory 21 (1995), no. 3, 376-381.

[7] Wolfram Research, Inc. Mathematica, Version 5.1, Wolfram Research, Inc., Champaign, IL, 1996.

MunMun Hazarika

Department of Mathematical Sciences

TEZPUR UNIVERSITY

NAPAM 784028, INDIA

E-mail address: munmun@tezu.ernet.in

Ambeswar Phukon

Department of Mathematics

Kokrajhar Govt. College

KoKRAJHAR-783370, INDIA

E-mail address: ambeswar@tezu.ernet.in 